\title{
Optical Logic Devices Based on Photonic Crystal
}

\author{
Kabilan Arunachalam ${ }^{1}$ and Susan Christina Xavier ${ }^{2}$ \\ ${ }^{1}$ Chettinad College of Engineering and Technology, Karur \\ ${ }^{2}$ Mookambigai College of Engineering, Pudukkottai
}

India

\section{Introduction}

Optical components that permit the miniaturization of photonic integrated circuits to a scale comparable to the wavelength of light are good candidates for future optical network and optical computing. All-optical communication is one of the solution for the electronic bottleneck viz speed and size, thanks to their ability to process the information at the speed of light. Optical logic gates are the fundamental components in optical digital information processing. In recent years, researchers have demonstrated all-optical logic gates using different schemes based on nonlinear effects in optical fibers (Ahn et al., 1997; Bogoni et al., 2005; Menezes et al., 2007), in semiconductor devices (Kyoung Sun Choi et al., 2010; Kim et al., 2002; Zhihong Li \& Guifang Li 2006; Dorren et al., 2004; Stubkjaer, 2000) and in waveguides (Tetsuro Yabu et al., 2002; Yaw-Dong Wu, 2005; Yaw-Dong Wu et al., 2008). But most of the reported works suffer from certain fundamental limitations including big size, low speed and difficult to perform chip-scale integration.

Nowadays, photonic crystals $(\mathrm{PhC})$ draw significant attention as a platform on which to build devices with dimensions in the order of wavelengths of light for future photonic integrated circuits. They are having some unique properties such as compactness, high speed, low power consumption, better confinement which make them promising candidate in photonic integrated circuits (Yablonovitch, 2003; Cuesta-Soto et al., 2004). Logic functions based on photonic crystal can be realized by nonlinear effect (Notomi et al., 2007), ring resonator (Andalib \& Granpayeh, 2009), and multimode interference (Hong-Seung Kim et al 2010). They require significant amount of power, nonlinear material, long interaction length and two different wavelengths for probing and input signals. One of the effects of complex spatial dispersion property in $\mathrm{PhC}$ namely self collimation provides a mechanism to employ optical switching and logic functions (Zhang et al., 2007; Susan et al., 2010).

\section{Theory of photonic crystal}

Photonic crystals $(\mathrm{PhC})$ are new class of optical material represented by natural or artificial structure with periodic modulation of the permittivity. Multiple interference of light on a periodic lattice leads to a photonic band gap and anomalous dispersion because light with a wavelength close to the period of modulation cannot propagate in certain directions. This 
peculiar property leads to an opportunity for a number of applications. Depending on the geometry of the structure, $\mathrm{PhC}$ can be classified into one-dimensional (1D), two dimensional (2D) and three-dimensional (3D) structures. Two-dimensional photonic crystals impose periodicity of the permittivity in two directions, while in the third direction the medium is uniform. Because of the ease of fabrication and analyzing, 2D photonic crystals have attractive attention of large number of researchers and engineers.

The properties of photonic crystal can be engineered through the process of doping which is achieved by either adding or removing dielectric material in a certain area. The dielectric materials then act as a defect region that can be used to localize an electromagnetic wave. Upon incident radiation, the periodic scatterers, that is the periodic dielectric materials in the photonic crystal could reflect an incident radiation at the same frequency in all directions. Wherever in space the reflected radiation interferes constructively, sharp peaks would be observed. This portion of the radiation spectrum is then forbidden to propagate through the periodic structure, and this band of frequencies is called photonic bandgap. On the other hand, wherever in space an incident radiation destructively interferes with the periodic scatterers in a certain directions, this part of the radiation spectrum will propagate through the periodic structure with minimal attenuation and this band of frequencies is called pass band. Introducing point defect or line defect, strict periodicity in the $\mathrm{PhC}$ is broken and can form optical cavity with high $\mathrm{Q}$ factor and low mode volume or lossless optical waveguide.

\subsection{Two dimensional square lattice and Brillouin zone}

In two dimensional photonic crystals the permittivity is modulated in two directions, say in the $\mathrm{x}$ and $\mathrm{z}$ plane: $\varepsilon(\mathrm{r})=\varepsilon(\mathrm{x}, \mathrm{z})$. Periodicity in two dimensions can be realized in various geometries, the most common being the square and the triangular lattices. In our work, we consider square lattice of silicon rods embedded in air background. This square lattice having a starting period $a^{\prime}=\sqrt{2} a$ along the $x$ axis where ' $a$ ' is lattice constant and oriented at $45^{\circ}$ with respect to the $x$ axis as shown in Figure 1a. Successive rods are shifted by $\delta x$ along the $\mathrm{x}$ axis and by $\delta \mathrm{z}$ along the $\mathrm{z}$ axis, $\delta \mathrm{x}$ and $\delta \mathrm{z}$ values are given as

$$
\delta x=\delta y=a^{\prime} / 2=a / \sqrt{2}
$$

In this case, the direct lattice is formed by the primitive vectors $a_{1}$ and $a_{2}$ in the real space and given by the following equations

$$
\begin{gathered}
a_{1}=a\left(\frac{\hat{x}}{\sqrt{2}}-\frac{\hat{z}}{\sqrt{2}}\right) \\
a_{2}=a\left(\frac{\hat{x}}{\sqrt{2}}+\frac{\hat{z}}{\sqrt{2}}\right)
\end{gathered}
$$

Where $\hat{\mathrm{x}}$ and $\hat{z}$ are unit vectors along the $\mathrm{x}$ axis and $\mathrm{z}$ axis respectively.

Reciprocal lattice vectors $b_{1}$ and $b_{2}$ in the reciprocal space are written as

$$
b_{1}=\frac{2 \sqrt{2} \pi \hat{x}}{a}
$$




$$
b_{2}=\frac{2 \sqrt{2} \pi \hat{z}}{a}
$$

The first Brillouin zone is defined as the region of the reciprocal space formed by the points which are closer to the origin than to any other vertex of the periodical lattice. Our structure has a diamond shaped Brillouin zone, which is illustrated in Figure 1d. The Irreducible Brillouin Zone (IBZ) is the triangular wedge in the bottom right corner and the rest of the Brillouin zone can be related to this wedge by rotational symmetry. The three special points $\Gamma, M$ and $X$ correspond to $(0,0),(\sqrt{2} \pi / a, 0)$ and $(\sqrt{ } 2 \pi / a, \sqrt{2} \pi / a)$ respectively. Due to periodicity structure, the behavior of the entire crystal can be obtained by studying the unit lattice in the IBZ.

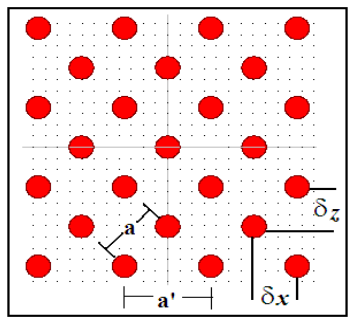

(a)

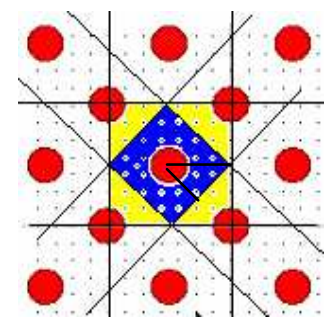

(c)

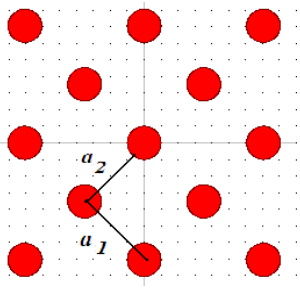

(b)

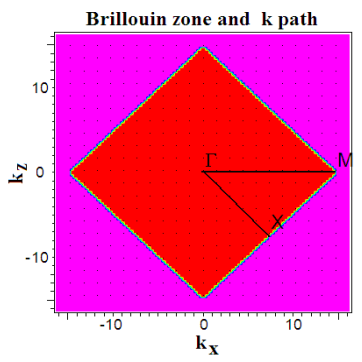

(d)

Fig. 1. a) Two-dimensional photonic crystal with square lattice b) primitive vectors $a_{1}$ and $a_{2}$ in real space c) \& d) First Brillouin Zone of square lattice

\subsection{Bloch-floquet theorem}

The dielectric function in wave vector is a function of spatial co-ordinates $\mathrm{r}$ and can be represented by

$$
\varepsilon(r)=\int g(k) e^{j k r} d k
$$

where $\mathrm{g}(\mathrm{k})$ is the dielectric function in wave vector representation and $\mathrm{k}$ is the wave vector.

In periodicity condition, the dielectric values of the function are repeated with lattice vector $\mathrm{R}$ in each direction and $\mathrm{R}=m \mathrm{a}_{1}+n \mathrm{a}_{2}$ where $m$ and $n$ are integers. The dielectric constant in a periodic structure is given as 


$$
\begin{gathered}
\varepsilon(r)=\varepsilon(r+R) \\
\varepsilon(r+R)=\int g(k) e^{j k r} e^{j k R} d k
\end{gathered}
$$

The propagation of a wave in a periodic medium is governed by the Bloch-Floquet theorem which is the product of a plane wave with a periodic function and states that

$$
E_{k}(r)=e^{j k r} u_{k}(r)
$$

where $u_{k}(r)$ is a periodic envelope function on the lattice and $u_{k}(r)=u_{k}(r+R)$.

\subsection{Photonic band gap - Plane Wave Expansion Method}

Photonic crystals have photonic band gap, which is the gap between the air-line and the dielectric line in the dispersion relation of PhC. Photonic crystals forbid the propagation of the range of frequency in the band gap, and allow the propagation of other frequencies with low loss. Photonic band diagram gives the information about the dispersion characteristics $w(\mathrm{k})$ for the Eigen mode of the PhC.

The Plane Wave Expansion method (PWE), can be used to calculate the band structure using an eigen formulation of the Maxwell's equations, and thus solving for the eigen frequencies for each of the propagation directions of the wave vectors (Igor A. Sukhoivanov \& Igor V. Guryev, 2009). The Helmholtz equations for TE and TM polarization can be derived from the fundamental Maxwell's equations,

$$
\begin{gathered}
\nabla \times\left[\nabla \times \frac{1}{\varepsilon_{r}(r)}\left(\varepsilon_{r}(r) E\right)\right]=\frac{\omega^{2}}{c^{2}}\left(\varepsilon_{r}(r) E\right) \\
\nabla \times\left[\frac{1}{\varepsilon_{r}(r)} \nabla \times H\right]=\frac{\omega^{2}}{c^{2}} H
\end{gathered}
$$

The dielectric function can be expanded to the Fourier series due to the periodicity

$$
\frac{1}{\varepsilon(r)}=\sum_{G} \chi(G) \cdot \exp (j G . r)
$$

where $G$ is a linear combination of reciprocal vector $G=l b_{1}+n b_{2}$ and $\chi(G)$ is Fourier expansion coefficient which depends on the reciprocal lattice vectors.

Substitution of Eq. 12 in Eq. 10 \& 11 gives

$$
\begin{aligned}
& \nabla \times\left[\nabla \times \sum_{G G^{\prime}} \chi\left(G-G^{\prime}\right) E\left(G^{\prime}\right) \exp (i(k+G) \cdot r)\right]= \\
& \frac{\omega^{2}}{c^{2}} \sum_{G} E(G) \exp (i(k+G) \cdot r)
\end{aligned}
$$




$$
\begin{aligned}
& \nabla \times\left[\nabla \times \sum_{G G^{\prime}} \chi\left(G-G^{\prime}\right) H\left(G^{\prime}\right) \exp (i(k+G) \cdot r)\right]= \\
& \frac{\omega^{2}}{c^{2}} \sum_{G} H(G) \exp (i(k+G) \cdot r)
\end{aligned}
$$

The eigen value equations for the Fourier expansion coefficients of electric field and magnetic field are

$$
\begin{aligned}
& \sum_{G} \chi\left(G-G^{\prime}\right)(k+G) \times\left[(k+G) \times E\left(G^{\prime}\right)\right]=-\frac{\omega^{2}}{c^{2}} E(G) \\
& \sum_{G} \chi\left(G-G^{\prime}\right)(k+G) \times\left[\left(k+G^{\prime}\right) \times H\left(G^{\prime}\right)\right]=-\frac{\omega^{2}}{c^{2}} H(G)
\end{aligned}
$$

These are 'Master Equations' for 2D photonic crystals. Here G and G' are in-plane reciprocal lattice vectors, $\mathrm{k}$ is in-plane wave vector and $\omega$ is the eigen frequency of the TE polarization. The $E(G)$ and $H(G)$ can be projected onto the unit and orthogonal vectors.

Rod type PhC consists of silicon dielectric rods with relative permittivity $\varepsilon_{a}$ periodically embedded in air with a dielectric permittivity $\varepsilon_{\mathrm{b}}$. For simplification, assume only one rod is present in the unit cell and the space dependence of the inverse of the permittivity $\chi$ in this elementary cell can be expressed as

$$
\frac{1}{\varepsilon_{r}}=\frac{1}{\varepsilon_{b}}+\sum_{R}\left(\frac{1}{\varepsilon_{a}}-\frac{1}{\varepsilon_{b}}\right) \theta(r-R)
$$

where $\theta(r)$ is the Heaviside function and its value is 1 inside the rod and 0 outside the rod and $\chi_{a}=1 / \varepsilon_{a}, \chi_{b}=1 / \varepsilon_{b}$. The expression for Fourier expansion coefficients of the dielectric function is represented by

$$
\chi(r)=\frac{1}{V_{O}} \int_{V_{O}} \frac{1}{\varepsilon_{r}} \exp (-j G r) d r
$$

where $V_{o}$ is the volume of the unit cell. In our structure, $V_{o}=a_{1} \hat{x} \times a_{2} \hat{z}+a_{1} \hat{z} \times a_{2} \hat{x}$. Substituting Eq. 17 in Eq. 18, the following equation can be obtained

$$
\chi(r)=\frac{1}{\varepsilon_{b}} \delta_{G, 0}+\frac{1}{V_{O}}\left(\frac{1}{\varepsilon_{a}}-\frac{1}{\varepsilon_{b}}\right) \int_{V_{O}} \theta(r) \exp (-j G r) d r
$$

where $\delta_{\mathrm{G}, 0}=1$ if $\mathrm{G}=0$ and $\delta_{\mathrm{G}, 0}=0$ if $\mathrm{G} \neq 0$. Using Bessel function Eq. 19 can be written as

$$
\chi(r)=\frac{1}{\varepsilon_{b}} \delta_{G, 0}+\left(\frac{1}{\varepsilon_{a}}-\frac{1}{\varepsilon_{b}}\right) \frac{2 \pi r_{a}^{2} J_{1}(G r)}{V_{o} G r}
$$

where $\pi r_{a}{ }^{2}$ is the cross-section area of the rod and $J_{1}(G r)$ is the first order Bessel function. The set of the reciprocal lattice vectors should now be selected to provide correct Fourier 
expansion of the dielectric function and the Bloch functions. Square lattice of silicon rod in air is considered in our structure with $\varepsilon_{\mathrm{a}}=11.56$ and $\varepsilon_{\mathrm{b}}=1$.

Thus from Eqs. 15 \& 16, for any given value for $\mathrm{k}$ leads to an infinite eigen value problem, these truncated by restricting $G$ to a set of $M$ vectors. The k-path within the first Brillouin zone are setting through $\Gamma, M$ and $X$ correspond to $(0,0),(\sqrt{2} \pi / a, 0)$ and $(\sqrt{2} \pi / a, \sqrt{2} \pi / a)$ respectively. The wave vector $\mathrm{k}$ describes the edge of the IBZ along the direction $\Gamma X, \Gamma \mathrm{M}$ and $\mathrm{MX}$ for reaching the extrema of $\omega(\mathrm{k})$ and this establishes the dispersion relation.

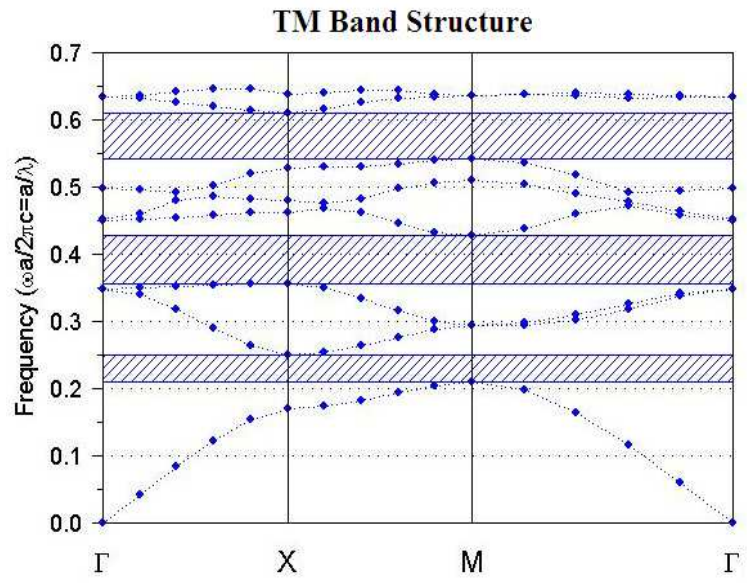

Fig. 2. Photonic band diagram of square lattice of silicon dielectric rod in the air

The Figure 2 shows the photonic band diagram of square lattice of silicon dielectric rod in the air. The radius of the silicon rod $\left(\mathrm{r}_{a}\right)$ is $0.3 a$. In this rod type, only TM polarization exists. The first band gap lies in the normalized frequency region $(\omega а / 2$ лс) 0.21 to 0.25 .

\subsection{Dispersion properties of photonic crystals}

Dispersion of the Bloch modes is one of the most important properties of the photonic crystals and it determines the propagation of modes in the crystal. It depends on many parameters of the $\mathrm{PhC}$ such as lattice type, the refractive index contrast between the dielectric material and the host material and distribution of atoms in the primitive cell.

Light pulse in a photonic crystal can be represented as a superposition of the Bloch modes with different Bloch vectors and frequencies

$$
u(r, t)=\sum_{m} \int f(k, m) \psi_{k, m}(r, t) d k
$$

where $\psi \mathrm{k}, \mathrm{m}$ is $m$-th Bloch mode with the Bloch vector $\mathrm{k}$ and $\mathrm{f}(\mathrm{k}, m)$ is the amplitude of the mode. The motion of the light pulse in photonic crystal is governed by the group velocity $v_{g}=\nabla_{k} . \omega(\mathrm{k})$. Since the light pulse is constructed from a superposition of several pulses with different combinations of $\mathrm{k}$ and $\mathrm{n}$, let us consider them independently. Each pulse component has the group velocity $v_{g, i}=\nabla_{k} . \omega(\mathrm{k}) \mid \mathrm{k}=\mathrm{k}_{i}$. If the group velocities $\mathrm{V}_{\mathrm{g}, \mathrm{i}}$ are 
close to each other the distortion of the original pulse will be minimal. If the group velocities are different the original pulse will widen. The group velocity and the group velocity dispersion are obtained from the dispersion diagram. The light propagation inside the PhC is governed by the Equifrequency Surface (EFS) which is the cross section of the band diagram at constant frequency. If the directions of the pulses components group velocities are perpendicular to the EFS, the widening of the original pulse is determined by the shape of the EFS. Each group velocity is locally perpendicular to local EFS. If the curvature of the EFS is large the original pulse will diverge or converge, depending on the sign of the curvature. So, depending on the EFS local curvature as well as on its evolution with the wavelength and the incident wave-vector, there are different types of effect are observed in PhC such as self-collimation, superlensing, negative refraction and superprism. These effects are used to control light propagation inside the $\mathrm{PhC}$.

\subsection{Self-collimation effect}

Self collimation effect is a linear non-diffraction phenomenon, totally independent of light intensity (Kosaka et al., 1999). PhC are designed to have dispersion properties that allow the beam to propagate without spatial spreading. In the equifrequency contour, flat square contour with zero curvature can be used to latterly confine the light since all the pulse components propagate with the same group velocity. This effect is called self-collimation effect. It provides a mechanism to control the light as in a waveguide.

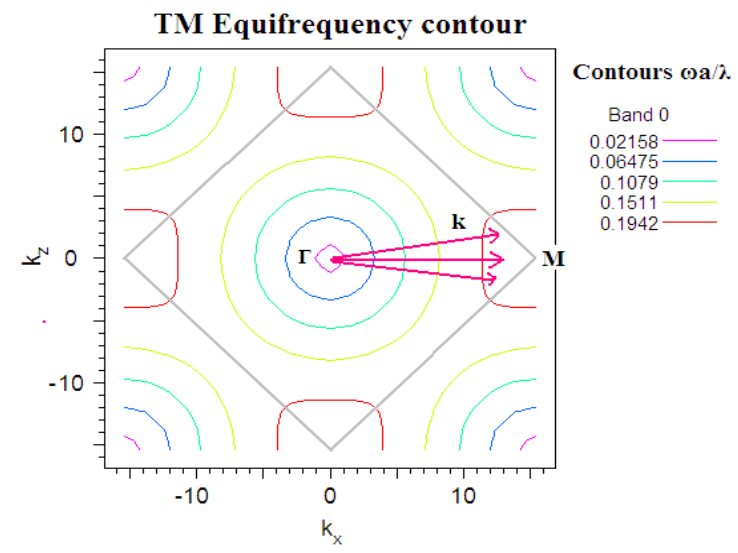

Fig. 3. First band TM Equifrequency contour of photonic crystal dispersion surface in the first Brillouin zone

The Figure 3 shows the frequency contour for the square lattice PhC consisting of silicon rods embedded in air with rod radius $0.3 a$. In this contour map, the curves of the frequencies around $0.194(a / \lambda)$ can be identified as squares with round corners centered at the M point, where $\lambda$ is the wavelength of incident radiation whose value is $1550 \mathrm{~nm}$. So self-collimation phenomenon occurs around the normalized frequency 0.194. RSOFT BandSOLVE tool is used to calculate band diagram and equifrequency contour. When light is incident from a high refractive index $\left(\mathrm{n}_{\mathrm{h}}\right)$ medium onto a low refractive index $\left(\mathrm{n}_{\mathrm{l}}\right)$ medium, the incident 
wave is totally reflected back into the high refractive index medium at the interface, provided the incident angle is larger than the critical angle given by $\theta c=\sin ^{-1}\left(n_{l} / n_{h}\right)$ (ChulSik Kee et al., 2007) . Self-collimated beams can be totally reflected at the interface of a PhC and air because $\mathrm{PhC}$ and air correspond to high refractive and low refractive mediums respectively. When it undergoes total internal reflection, the field amplitude decays very rapidly into air and becomes negligible at a distance within one lattice constant. An air layer created by introducing a line defect by removing a few rods in a row is expected to give rise to total internal reflection. Reflection provides a mechanism for bending and splitting of self-collimated beams.

\section{All optical logic gates}

All-optical logic gates will be the key elements used in next generation optical computer and optical network. All-optical signal processing can handle large bandwidth signals, large information flows and no need of electrical to optical conversion. All-optical logic gates are capable of performing many logic functions. These are expected to find many applications in optical communication, photonic microprocessors, optical signal processors, optical instrumentation, etc. AND logic gate is used to perform address recognition, packet-header modification, and data-integrity verification. All-optical AND-gates have served as sampling gates in optical sampling oscilloscopes (Westlund, et al., 2005) owing to their ultrafast operation compared to traditional electrical methods. XOR gates can perform a diverse set of processing functions, including comparison of data patterns for address recognition and subsequent packet switching, optical generation of pseudorandom patterns, data encryption/decryption, and parity checking. Threshold detector functionality can be realized by XNOR logic gate. All-optical NOT-gates can be used as inverter and switches. Combination of logic gates may be employed to perform basic or complex computing and arithmetic functions such as binary addition, subtraction, comparison, decoding, encoding and flipflops.

\subsection{Structure and optimum values of the proposed logic gates}

The proposed logic gate is a square lattice two dimensional $\mathrm{PhC}$ that consists of silicon dielectric rods in air background. The structure has a width of $23 \sqrt{2} a$ in x-direction and a length of $25 \sqrt{2} a$ in $z$-direction. The dielectric constant and the refractive index of the dielectric rods are 12.0 and 3.46 respectively. In this structure the square lattice is oriented at $45^{\circ}$ with the interface parallel to the $\Gamma$-M direction with period $a^{\prime}=\sqrt{2} a$ where ' $a$ ' is a lattice constant. Successive cells are shifted by $\delta x$ along the $x$ axis and $\delta z$ along the $z$ axis. The amount of shifting is $\delta \mathrm{x}=\delta \mathrm{z}=a^{1 / 2}=\sqrt{2} a / 2$. The Figure $4 \mathrm{a}, 4 \mathrm{~b}$ and $4 \mathrm{c}$ illustrate the $2 \mathrm{D}$ $\mathrm{PhC}$ lattice used for designing logic gates. The circles represent the silicon rods whose radii are $\mathrm{r}=0.35 a=105 \mathrm{~nm}$, where $a=302 \mathrm{~nm}$ is the lattice constant.

The schematic circuital layout of the proposed logic gates is shown in Figure 4d. Electrical input signals 1 and 2 activate synchronized laser light sources 1 and 2 respectively to generate optical input signals $I_{1}$ and $I_{2}$. The reference signal $I_{\text {ref }}$ is obtained from reference laser phase locked with lasers 1 and 2 . All the input signals including reference have same frequency, polarization, phase, and optical path, with only the reference signal having different amplitude for different gate types. Two input optical signals are coupled using $Y$ coupler and applied to one of the input port of the crystal device and the reference signal 
$\mathrm{I}_{\mathrm{ref}}$ is launched at the second input port. The photonic crystal structure with mirror and splitter performs a specific logic gate function by combining the reflected signal and the partially transmitted reference signal. The optical output is detected and converted into electrical signal by photo detector. This structure can be used for stand alone logic gates. In an integrated circuit the output value will be standardized using a PhC amplifier and given to the input port of the next in sequence logic gate and so on.

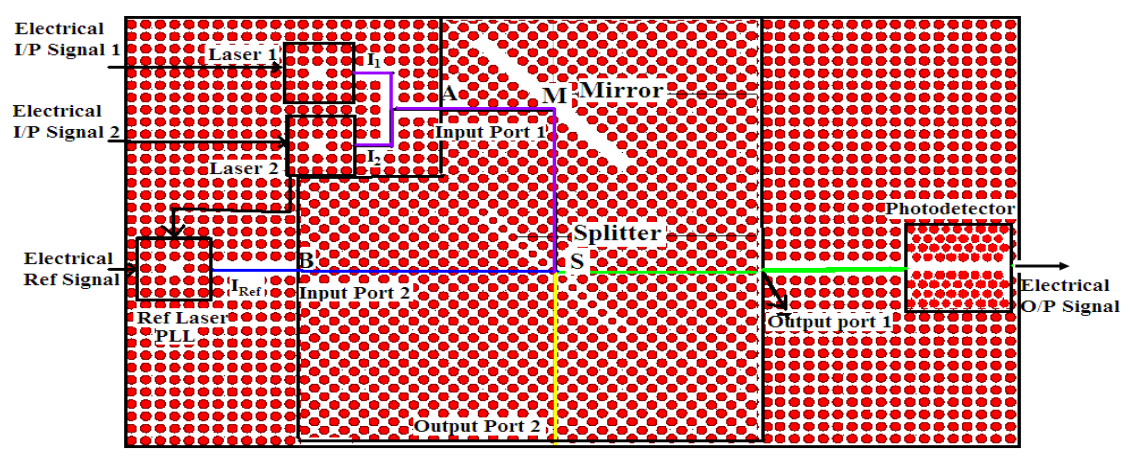

Fig. 4. a Proposed structure of AND, NAND, NOR \& XNOR logic gates

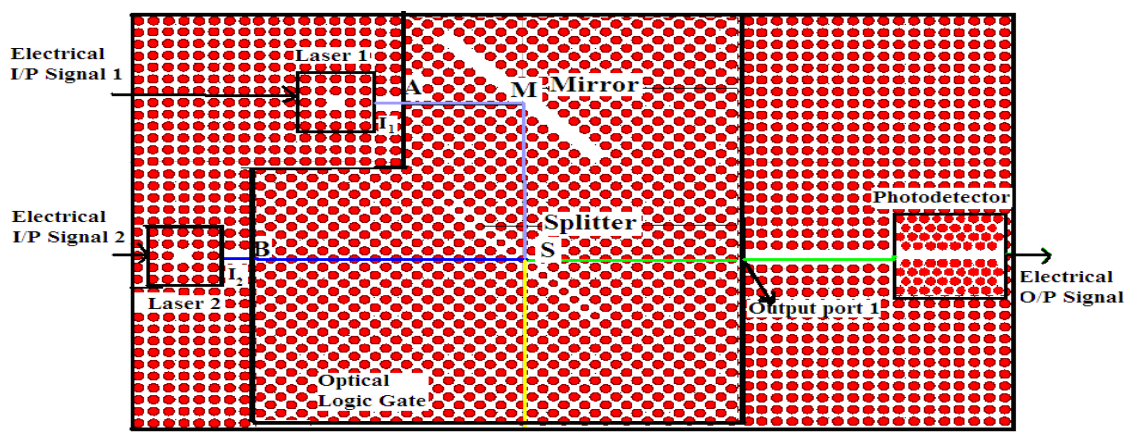

Fig. 4. $\mathrm{b}$ Proposed structure of XOR logic gate

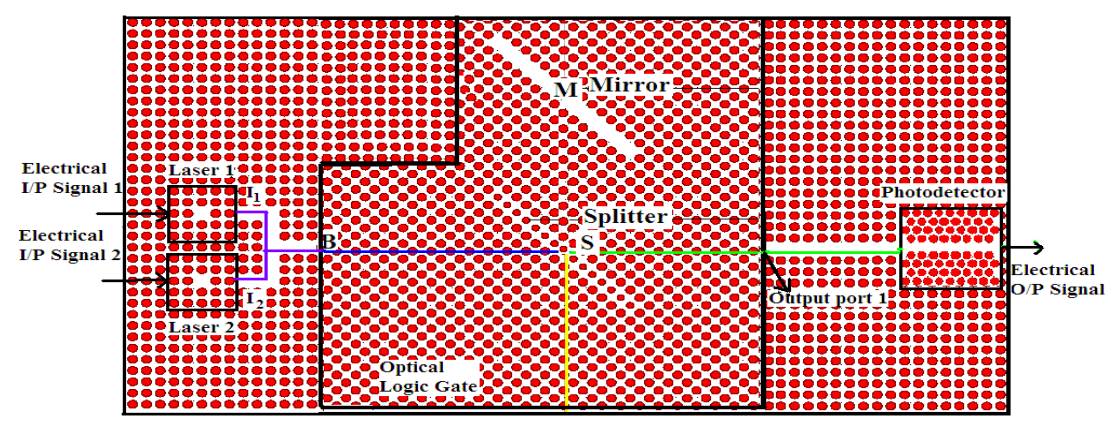

Fig. 4. c Proposed structure of OR logic gate 


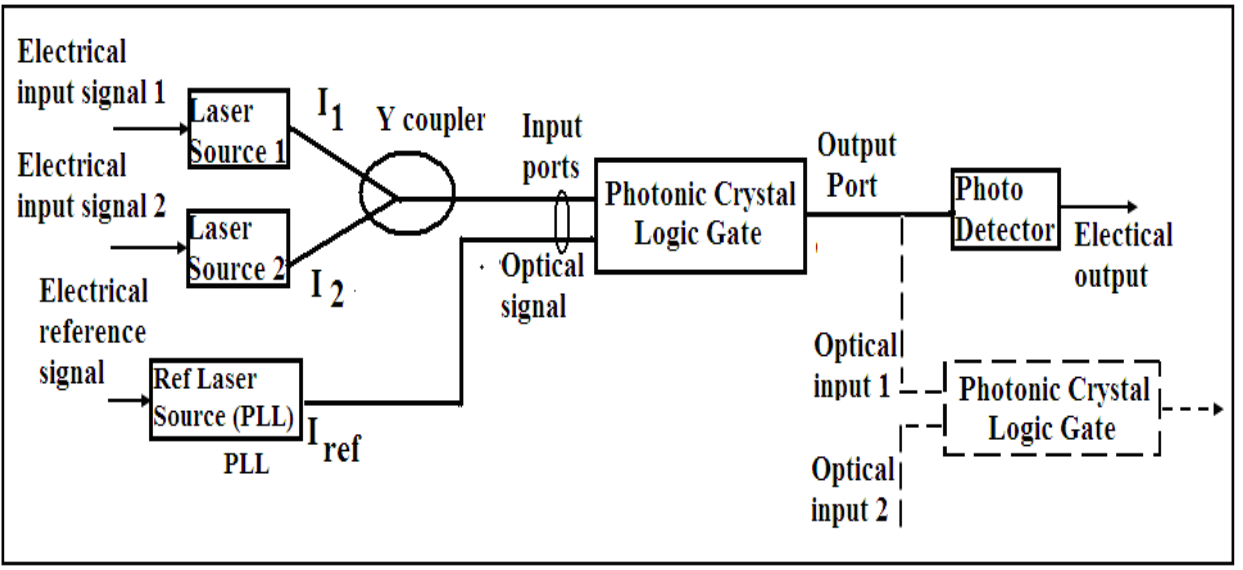

Fig. 4. d Schematic circuital layout of the proposed logic gates

The proposed photonic crystal based logic gate utilizes both bending and splitting mechanisms of self collimating beam. In this structure two line defects are created by reducing the radius of 15 rods in the $\Gamma-X$ direction. First line defect, in which 15 rods are completely removed act as mirror $(\mathrm{M})$ and second one, in which the radius of the defect rods are reduced act as a splitter (S). When the self collimated beam is incident at rod-air interface, it is partially reflected and partially transmitted and there is a phase change ' $\varnothing$ ' occurs in the reflected wave. The power splitting ratio at the line defect and phase difference between the transmitted and reflected signals are dependent on the radius of the rod. From the Figure 5 it is evident that at the defect rod radius $r_{d}=83 \mathrm{~nm}=0.274 a$ the transmitted and the reflected powers are divided equally and at $r_{d}=0$ the mirror completely reflects the incident beam. Thus mirror completely reflects the incident beam and the splitter splits the beam with the power ratio 50:50.

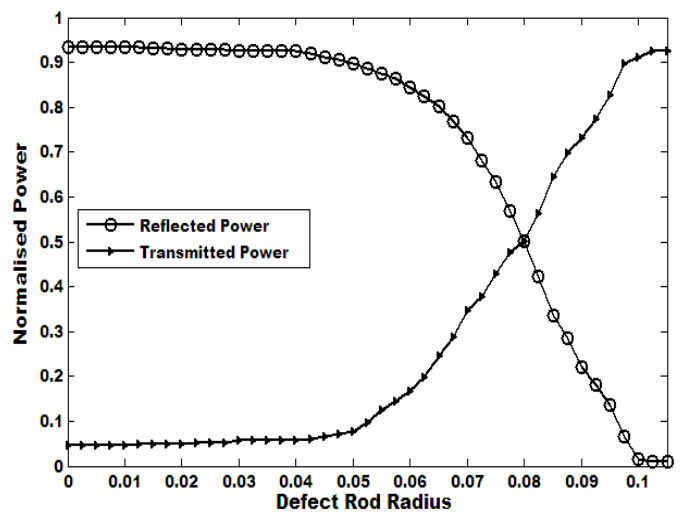

Fig. 5. Normalized transmitted and reflected power with respect to defect rod radius

The phase difference between the transmitted and reflected beam depends on the defect rod radius. If the defect rods radii $r_{d}$ varied and greater than host rods radii the phase 
difference between the transmitted and the reflected signal is $-\Pi / 2$ and if it is less than host rods radii the phase difference is $\Pi / 2$ (Deyin Zhao et al., 2007).

PhC logic gate structure consists of four ports. Inputs and reference inputs are applied to port 1 and port 2 and the outputs are taken from the output ports 1 and 2. All incident signals including reference signal having the same wavelength of $1.55 \mu \mathrm{m}$, phase, and polarization. At the interfering point the path lengths of input signals are equal that is the path length from the AMS is equal to the path length BS which is set as $16 \sqrt{2} a$. The reflected input signal from the mirror interferes with another signal at the splitter. This interference is either constructive or destructive depending on their phase difference. The output taken from the output port 1 is destructive and port 2 is constructive.

Based on the relation between the transmitting and the reflecting signal $t^{2}+r^{2}=1$, the transmission amplitude of the beam is $\mathrm{e}^{i \theta} / \sqrt{2}$ and the reflection amplitude is $\mathrm{e}^{i\left(\theta+\frac{\pi}{2}\right)} / \sqrt{2}$.The propagation of a wave in a periodic medium is governed by the FloquetBloch theorem which is given by $E(r)=u(r) \mathrm{E} e^{-i \phi}$, where $\mathrm{E}$ is a plane wave and $u(r)$ has the periodicity of the $\mathrm{PhC}$. The transmitted and reflected signals are expressed as

$$
\begin{aligned}
& R_{12}=\frac{u \mathrm{E}}{\sqrt{2}} e^{i \phi_{1}} \\
& T_{22}=\frac{u \mathrm{E}}{\sqrt{2}} e^{i \phi_{2}}
\end{aligned}
$$

where $R_{12}$ is reflected input signal 1 at splitter and $T_{22}$ is the transmitted input signal 2 and $\Phi_{1}$ and $\Phi_{2}$ are the phase shifts of reflected and transmitted signals respectively. The resultant signal at the output port is a linear combination of reflected and transmitted beam, expressed as

$$
O=R_{12}+T_{22}=\frac{u E}{\sqrt{2}}\left(e^{i \phi_{1}}+e^{i \phi 2}\right)
$$

and its corresponding intensity

$$
I=|O|^{2}=\left|\frac{u \mathrm{E}}{\sqrt{2}}\left(e^{i \phi_{1}}+e^{i \phi_{2}}\right)\right|^{2}
$$

Intensity of the reference signal is set at different levels according to the desired logic gates. In our simulations the mesh sizes in the $x$ - and $z$-directions are set to be $a / 16$. The time step for this mesh size is calculated from the Eq. 26

$$
\nabla t=\frac{1}{c \sqrt{\frac{1}{\nabla x^{2}}+\frac{1}{\nabla y^{2}}}}
$$


and it is found to 0.04 femtosecond. The calculated area is surrounded by a Perfectly Matched Layer (PML) boundary.

\subsection{Realization and simulation results of logic gates}

\subsubsection{XOR and OR logic gates}

The input signals $I_{1}$ and $I_{2}$ are applied to the port 1 and 2 and the XOR gate outputs is taken from the output port $1 . I_{1}$ and $I_{2}$ are equal to $P_{0}$. The reflected input signal $I_{1}$ from the mirror interfered with either reflected signal or transmitted signal of $\mathrm{I}_{2}$ at the splitter. Either destructive or constructive interference are obtained at port 1 or 2 respectively depending on their phase difference. When both the input signals are same the output of XOR gate is zero and both are different the output is one.

In the case of OR gate, the input signals are combined and applied to the input port 2 and the output is taken from the output port 1 . When both the signals are zero the output is zero and if any one of the input is high, the output is also high. To validate this theoretical prediction, steady state electromagnetic field distribution is simulated using FDTD method. Rsoft FullWAVE software is used to simulate logic gate functions. The field distributions of XOR and OR logic gates are shown in Figure 6 and their corresponding truth table is shown in Table 1.

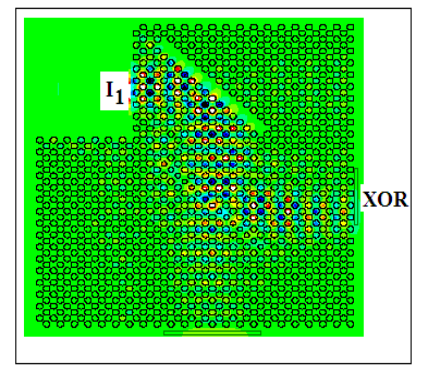

(a)

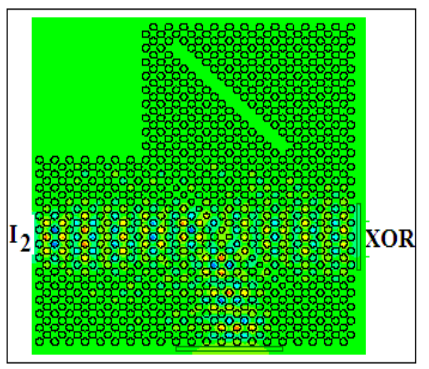

(b)

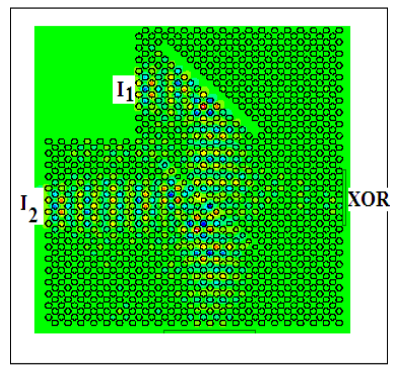

(c)

Fig. 6. Electromagnetic field distributions of XOR logic gates. (a) \& (b) any one of the input signal is applied (c) both input signals are excited simultaneously

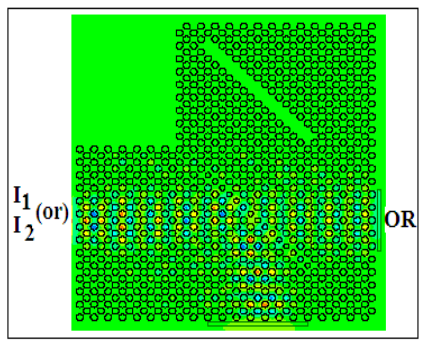

(a)

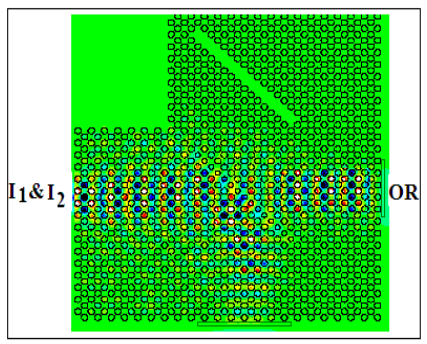

(b)

Fig. 7. Field distributions of OR logic gates. a) any one of the input signal is applied b) both input signals are excited simultaneously 


\begin{tabular}{|c|c|c|c|c|c|}
\hline \multirow[b]{2}{*}{$\mathrm{I}_{1}$} & \multirow[b]{2}{*}{$\mathrm{I}_{2}$} & \multicolumn{2}{|c|}{ XOR } & \multicolumn{2}{|c|}{ OR } \\
\hline & & $\mathrm{O} / \mathrm{P}$ power & Logic level & $\mathrm{O} / \mathrm{P}$ power & Logic level \\
\hline 0 & 0 & 0 & 0 & 0 & 0 \\
\hline 0 & 1 & $0.5 \mathrm{P}_{0}$ & 1 & $0.5 \mathrm{P}_{0}$ & 1 \\
\hline 1 & 0 & $0.5 \mathrm{P}_{0}$ & 1 & $0.5 \mathrm{P}_{0}$ & 1 \\
\hline 1 & 1 & $0.001 \mathrm{P}_{0}$ & 0 & $1.00 \mathrm{P}_{0}$ & 1 \\
\hline
\end{tabular}

Table 1. Truth table for XOR and OR gate

\subsubsection{AND logic and NOR logic gates}

To realize AND logic, inputs $\mathrm{I}_{1}, \mathrm{I}_{2}$ and reference beam $\mathrm{I}_{\text {ref }}$ are taken as $\mathrm{P}_{0}$ and $0.5 \mathrm{P}_{0}$ and combined inputs $I_{1}$ and $I_{2}$ are applied at input port 1 and $I_{\text {ref }}$ is applied at port 2 . The destructive interfered signal at the output port 1 is considered as a AND output. In AND logic, when both the input signals are high the output is high otherwise it is zero. In simulation it is found that the output intensity is $0.24 \mathrm{P}_{0}$ for separate excitation of $\mathrm{I}_{1}$ and $\mathrm{I}_{2}$ and $0.751 \mathrm{P}_{0}$ for simultaneous excitation of both $\mathrm{I}_{1}$ and $\mathrm{I}_{2}$. If both the signals are not applied the output is $0.25 \mathrm{P}_{0}$.In the case of NOR gate, inputs $\mathrm{I}_{1}, \mathrm{I}_{2}$ and reference beam $\mathrm{I}_{\text {ref }}$ are considered as $\mathrm{P}_{0}$ and $1.5 \mathrm{P}_{0}$ respectively. The logic function of this gate is, if both the inputs are low the output is high or else it is zero. The simulation results show that when both the input signals are zero the output is $0.759 \mathrm{P}_{0}$ and if any one signal is applied the output is $0.25 \mathrm{P}_{0}$. Truth table for AND logic and NOR logic gates is tabulated in Table 2.

\begin{tabular}{|c|c|c|c|c|c|}
\hline \multirow{2}{*}{$\mathrm{I}_{1}$} & \multirow{2}{*}{$\mathrm{I}_{2}$} & \multicolumn{2}{|c|}{$\mathrm{AND}\left(\mathrm{I}_{\mathrm{ref}}=0.5 \mathrm{P}_{0}\right)$} & \multicolumn{2}{c|}{ NOR $\left(\mathrm{I}_{\mathrm{ref}}=1.5 \mathrm{P}_{0}\right)$} \\
\cline { 3 - 6 } & & $\mathrm{O} / \mathrm{P}$ power & Logic level & $\mathrm{O} / \mathrm{P}$ power & Logic level \\
\hline 0 & 0 & $0.25 \mathrm{P}_{0}$ & 0 & $0.759 \mathrm{P}_{0}$ & 1 \\
\hline 0 & 1 & $0.24 \mathrm{P}_{0}$ & 0 & $0.25 \mathrm{P}_{0}$ & 0 \\
\hline 1 & 0 & $0.24 \mathrm{P}_{0}$ & 0 & $0.25 \mathrm{P}_{0}$ & 0 \\
\hline 1 & 1 & $0.751 \mathrm{P}_{0}$ & 1 & $0.25 \mathrm{P}_{0}$ & 0 \\
\hline
\end{tabular}

Table 2. Truth table for AND logic and NOR gate

Steady state electromagnetic field distributions of NOR gate and AND gate for various input combinations are shown in Figure 8 and Figure 9 respectively.

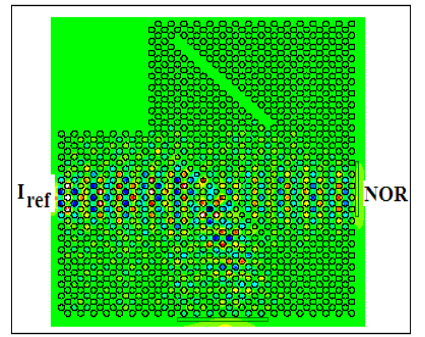

(a)

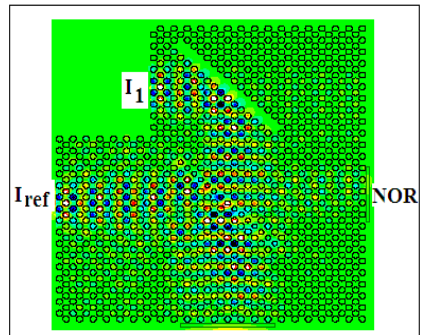

(b)

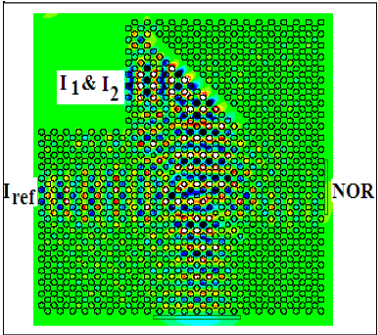

(c)

Fig. 8. Electromagnetic field distributions of NOR logic gates. (a) no input signal is applied (b) any one of the input signal is applied c) both input signals are applied simultaneously 


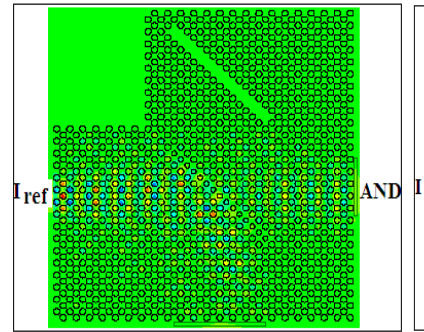

(a)

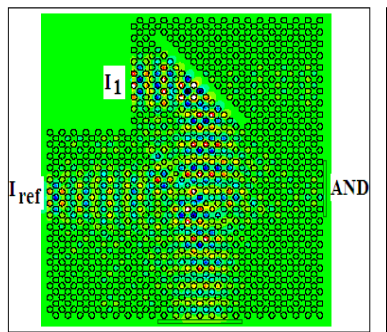

(b)

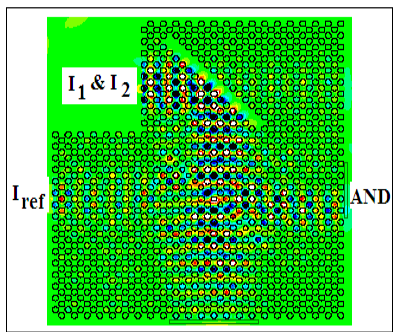

(c)

Fig. 9. Electromagnetic field distributions of AND logic gates. a) both the input signals are low b) any one of the input signal is high c) both input signals are high.

\subsubsection{NAND and XNOR logic gates}

For NAND logic realization, inputs $I_{1}$ and $I_{2}$ are set as $P_{0}$ and reference beam $I_{\text {ref }}$ is set as $2.5 \mathrm{P}_{0}$. These are applied at input port 1 and 2 respectively. In NAND logic, when both the input signals are high the output is zero and any one of the input signal is low the output is high. It is evident from the simulation that when none of the signal is applied the output is $1.25 \mathrm{P}_{0}$ and if any one of the signal is applied the output is $0.756 \mathrm{P}_{0}$. If both the signals are excited the output is $0.25 \mathrm{P}_{0}$. The inputs $\mathrm{I}_{1} \& \mathrm{I}_{2}$ and $\mathrm{I}_{\mathrm{ref}}$ are considered as $\mathrm{P}_{0}$ for XNOR gate realization. Logic operation for XNOR gate is known that when both the inputs are same the output is high and if both the inputs are different the output is low. In simulation, it is found that the output is $0.505 \mathrm{P}_{0}$ for simultaneous excitation of inputs and also for none of the input signal. When any one of the signal is applied the output is $0.001 \mathrm{P}_{0}$. The simulated field distributions of XNOR logic gate are illustrated in Figure 10 and NAND logic gate field distribution is shown in Figure 11. Table 3 explicates the truth table for NAND and XNOR logic gates.

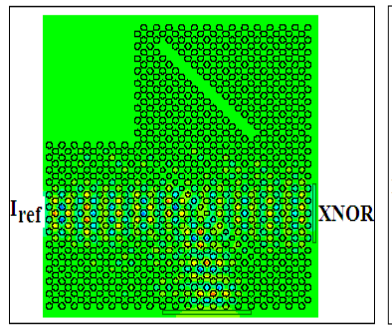

(a)

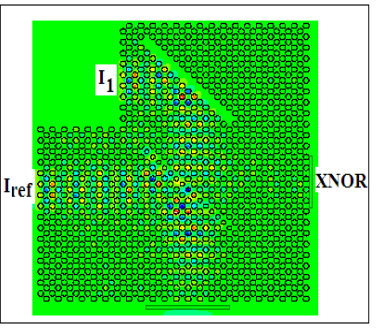

(b)

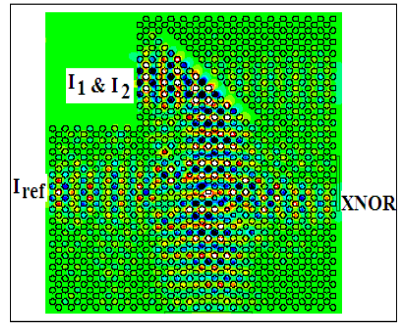

(c)

Fig. 10. Electromagnetic field distributions of XNOR logic gates. (a) both the input signals are low b) any one of the input signal is applied (c) both the input signals are applied simultaneously. 


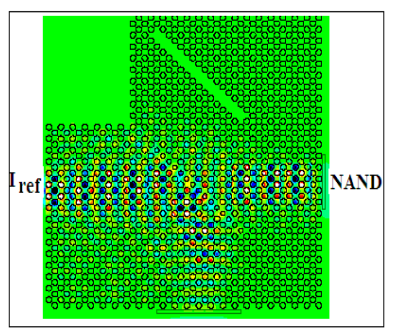

(a)

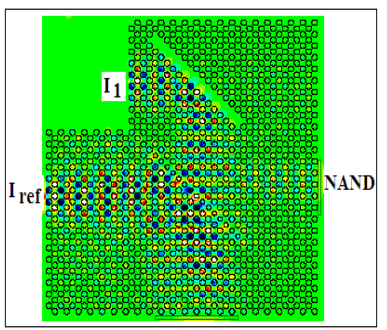

(b)

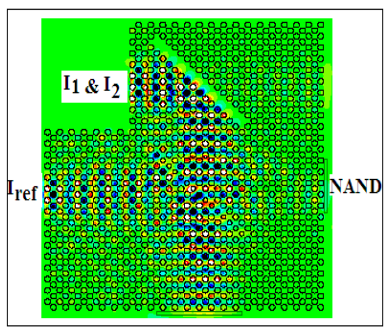

(c)

Fig. 11. Field distribution of NAND logic gates a) no input is applied b) any one of the input signal is excited c) both the signals are applied simultaneously

\begin{tabular}{|c|c|c|c|c|c|}
\hline \multirow{2}{*}{$\mathrm{I}_{1}$} & \multirow{2}{*}{$\mathrm{I}_{2}$} & \multicolumn{2}{|c|}{ NAND $\left(\mathrm{I}_{\text {ref }}=2.5 \mathrm{P}_{0}\right)$} & \multicolumn{2}{c|}{ XNOR $\left(\mathrm{I}_{\text {ref }}=\mathrm{P}_{0}\right)$} \\
\cline { 3 - 6 } & & $\mathrm{O} / \mathrm{P}$ power & Logic level & $\mathrm{O} / \mathrm{P}$ power & Logic level \\
\hline 0 & 0 & $1.25 \mathrm{P}_{0}$ & 1 & $0.505 \mathrm{P}_{0}$ & 1 \\
\hline 0 & 1 & $0.756 \mathrm{P}_{0}$ & 1 & $0.001 \mathrm{P}_{0}$ & 0 \\
\hline 1 & 0 & $0.756 \mathrm{P}_{0}$ & 1 & $0.001 \mathrm{P}_{0}$ & 0 \\
\hline 1 & 1 & $0.25 \mathrm{P}_{0}$ & 0 & $0.505 \mathrm{P}_{0}$ & 1 \\
\hline
\end{tabular}

Table 3. Truth table for NAND logic and XNOR logic gate

\section{Photonic crystal optical logic devices for a packaged system}

In a complete packaged system, photonic crystal based laser light sources, logic gates and detector are integrated within a single chip. Figure 12 illustrates the integrated photonic crystal based devices. Light source laser is based on a 2D photonic crystal slab patterned with a square lattice. Holes are drilled in GaAs dielectric material. The periodicity of the holes is fixed at $315 \mathrm{~nm}$, and the hole radius is tuned from 105 to $130 \mathrm{~nm}$ to change the resonance frequency of the cavities (Hatice Altug et al., 2006). Lasers are driven by the given electrical signals and the corresponding optical output is applied to the all-optical logic gate. Laser $1 \& 2$ output signals are coupled using coupler and launched to the input port 1 of logic gate and the phase locked reference signal is applied to input port 2. All-optical logic gate performs the logical functions in optical domain.

In the integrated photonic crystal based logic gates, output value of the logic gate will be standardized using a PhC amplifier. The gain of the amplifier is adjusted such that the output level is either " 1 " or " 0 ". In Figure 12 AND logic gate output is standardized and given to one of the input of XOR gate and other input is getting from the output of another preceding logic gate. Thus the standardized output of one logic gate is given to the input port of the next in sequence logic gate and so on. Finally the output of the last logic gate is applied to the photodetector. The photodetector detects the optical signal. Photo detector is designed using triangular air-hole photonic crystal with lattice constant is 420nm and slab thickness is $204 \mathrm{~nm}$ (M Notomi and T Tanabe 2010). The detector converts the optical signal into electrical output. 


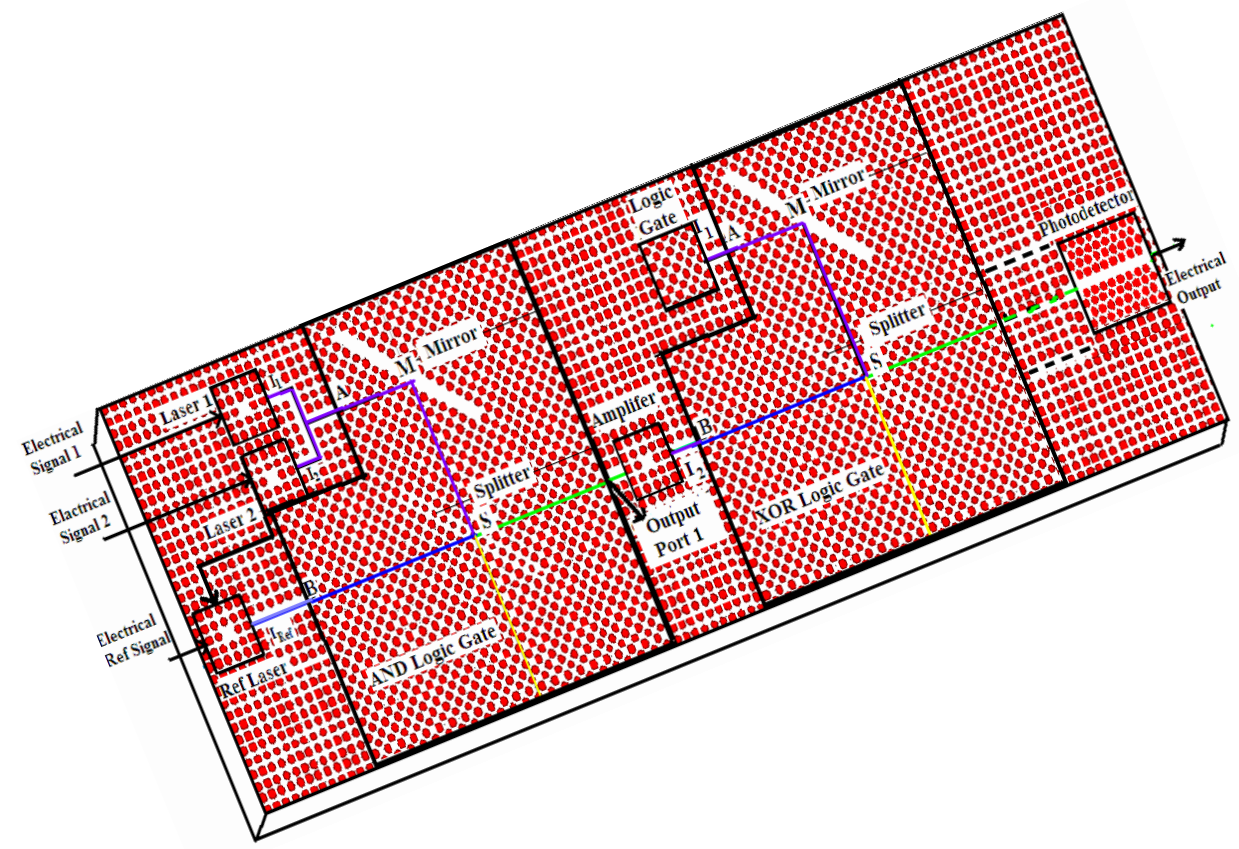

Fig. 12. Integrated photonic crystal based devices

\section{Challenges in fabrication}

The designing of the beam splitter and the mirror requires high precision of fabrication. Any small deviation from the design of the beam splitters leads to a decay of the optical performance. For instance, a beam splitter would present a wrong phase shift, optical loss, unequal splitting or even mirror like operation if its narrow veins are unintentionally narrower or removed. The Silicon rods must be uniform, smooth and vertical at the side wall in order to maintain the collimation effect throughout the device. The optical path length of the interfering signals should be maintained equal otherwise it will lead to additional phase shift.

\section{Conclusion}

Thus all the logic gates functions are implemented in non-channel photonic crystal. Selfcollimation, bending and splitting effects are used to realize logic gates. The Finite Difference Time Domain method (FDTD) gives fairly accurate results in line with the theoretically predicted concepts. The proposed design exhibits an on-off contrast ratio around $3 \mathrm{~dB}$ and a device size of $10 \times 10 \mu \mathrm{m}^{2}$ operating at the optical communication wavelength $1550 \mathrm{~nm}$. The main advantages of all-optical logic gates are small dimensions, simple structure and high speed. These devices may turn out to be good candidate for optical computing and photonic integrated circuits. 


\section{References}

Ahn, K. H.; Cao, X. D. ; Liang, Y.; Barnett, B. C. ; Chaikamnerd, S. \& Islam M. N. (1997). Cascadability and functionality of all-optical low-birefringent non-linear optical loop mirror: Experimental demonstration, Journal Optical Society of America B, Vol. 14, No. 5, pp. 1228-1236

Andalib, P. \& Granpayeh,N. (2009). All-optical ultra-compact photonic crystal AND gate based on nonlinear ring resonators, Journal Optical Society of America B, Vol. 26, No.1, pp. 10-16

Bogoni, A. ; Poti 'L.; Proietti, R.; Meloni, G. ; Ponzini, F. \& Ghelfi, P.(2005). Regenerative and reconfigurable all-optical logic gates for ultra-fast applications, Electronics Letters, Vol. 41, No. 7, pp. 435-436

Chul-Sik Kee; Do-Kyeong Ko; Jongmin Lee; Sun-Goo Lee \& Hae Yong Park (2007). SelfCollimated Beams in Two-Dimensional Photonic Crystals: Properties and Applications, Journal of the Korean Physical Society, Vol. 51, No. 4, pp. 1479-1483

Cuesta-Soto, F.; Martínez, A.; García, J.; Ramos, F.; Sanchis, P.; Blasco, J. \& Martí, J. (2004). All-optical switching structure based on a photonic crystal directional coupler, Optics Express, Vol. 12, No. 1, pp. 161-167

Deyin Zhao; Jie Zhang; Peijun Yao \& Xunya Jiang (2007). Photonic crystal Mach-Zehnder interferometer based on self-collimation, Applied Physics Letter, Vol. 90, pp. 231114231116

Dorren, H. J. S. ; Xuelin Yang ; Mishra, A.K.; Zhonggui Li ; Heongkyu Ju ; De Waardt, H.; Khoe, G.D.; Simoyama, T. ; Ishikawa, H. ; Kawashima, H. \& Hasama, T. (2004). All-optical logic based on ultrafast gain and index dynamics in a semiconductor optical amplifier, IEEE Journal of Selected Topics Quantum Electronics, Vol. 10, No. 5, pp. 1079-1092

Hatice Altug ; Dirk Englund and Jelena Vuckovic(2006). Ultrafast photonic crystal nanocavity laser, Nature Physics, Vol 2, pp. 484-488

Hong-Seung Kim; Tae-Kyung Lee ; Geum-Yoon Oh ; Doo-Gun Kim \& Young-Wan Choi (2010). Analysis of all optical logic gate based on photonic crystals multimode interference, Proceedings of SPIE, ISBN 9780819480026, San Francisco, 23-28 January 2010

Igor A. Sukhoivanov \& Igo r V. Guryev (2009), Photonic Crystals Physics and Practical Modeling, Springer Series in optical sciences, ISBN 978-3-642-02645-4, USA

Kim, J.H.; Jhon, Y.M. ; Byun, Y.T. ; Lee, S. ; Woo, D.H. \& Kim, S.H. (2002). All-optical XOR gate using semiconductor optical amplifiers without additional input beam, IEEE Photonics Technology Letters, Vol.14, No.10, pp. 1436-1438

Kosaka, H. ; Kawashima, T. ; Tomita, A.; Notomi, M. ; Sato, T. \& Kawakami ,S. (1999) Selfcollimating phenomena in photonic crystals, Applied Physics Letter. Vol. 74, pp. 1212-1214

Kyoung Sun Choi; Young Tae Byun; Seok Lee \& Young Min Jhon (2010). All-Optical OR/NOR Bi-functional Logic Gate by Using Cross-gain Modulation in semiconductor Optical Amplifiers, Journal of the Korean Physical Society, Vol. 56, No. 4, pp. 1093-1096

Menezes, J. W. M.; Fraga, W. B.; Ferreira, A. C.; Saboia, K. D. A.; Filho, A. F. G. F.; Guimarães,G. F.; Sousa, J. R. R.; Rocha, H. H. B.\& Sombra, A. S. B.(2007). Logic 
gates based in two- and three-modes nonlinear optical fiber couplers, Optical and Quantum Electronics, Vol. 39, No. 14, pp. 1191-1206

Notomi, M. ; Tanabe, T.; Shinya, A. ; Kuramochi, E. ; Taniyama, H.; Mitsugi, S. \& Morita, M.(2007). Nonlinear and adiabatic control of high-Q photonic crystal nanocavities, Optics Express, Vol. 15, No.26, pp. 17458-17481

Notomi, M. \& Tanabe, T. (2007). Pure-crystal-silicon detector has ultralow dark current, SPIE Newsroom, DOI: 10.1117/2.1201004.002936

Stubkjaer, K. E. (2000). Semiconductor optical amplifier-based all-optical gates for highspeed optical processing, IEEE Journal of Selected Topics in Quantum Electronics, Vol. 6, No. 6, pp. 1428-1435

Susan Christina,X. ; Kabilan, A.P. \& Elizabeth Caroline,P. (2010). Ultra Compact All Optical Logic Gates Using Photonic Band-Gap Materials, Journal of Nanoelectronics and Optoelectronics, Vol. 5, No.3, pp. 397-401

Tetsuro Yabu; Masahiro Geshiro; Toshiaki Kitamura; Kazuhiro Nishida \& Shinnosuke Sawa (2002). All-Optical Logic Gates Containing a Two-Mode Nonlinear Waveguide, IEEE Journal of Quantum Electronics, Vol. 38, No. 1, pp. 37-46

Westlund, M.; Andrekson, P. A.; Sunnerud, H.; Hansryd, J. and Li, J. (2005), High Performance Optical-Fiber-Nonlinearity-Based Optical Waveform Monitoring, IEEE Journal Lightwave Technology, Vol 23, pp. 2012 - 2022

Yablonovitch, E. (2003). Photonic bandgap based designs for nano-photonic integrated circuits, Proceedings of International Semiconductor Device Research Symposium, ISBN 0-7803-8139-4, Washington, December 10-12,2003

Yaw-Dong Wu (2005). All-Optical Logic Gates by Using Multibranch Waveguide Structure With Localized Optical Nonlinearity, IEEE Journal of Selected Topics in Quantum Electronics, Vol. 11, No. 2, pp. 307-312

Yaw-Dong $\mathrm{Wu}$; Tien-Tsorng Shih \& Mao-Hsiung Chen (2008). New all-optical logic gates based on the local nonlinear Mach-Zehnder interferometer, Optics Express , Vol. 16, No. 1, pp. 248-257

Zhang, Y. L. ; Zhang, Y. \& Li, B. J. (2007). Optical switches and logic gates based on selfcollimated beams in two-dimensional photonic crystals, Optics Express, Vol. 15, No.15, pp. 9287-9292

Zhihong Li \& Guifang Li (2006). Ultrahigh Speed Reconfigurable Logic Gates Based on Four-Wave Mixing in a Semiconductor Optical Amplifier, IEEE Photonics Technology Letters, Vol. 18, No. 12, pp. 1341-1343 


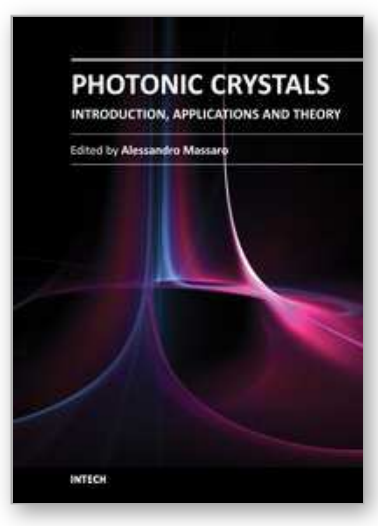

\author{
Photonic Crystals - Introduction, Applications and Theory \\ Edited by Dr. Alessandro Massaro
}

ISBN 978-953-51-0431-5

Hard cover, 344 pages

Publisher InTech

Published online 30, March, 2012

Published in print edition March, 2012

The first volume of the book concerns the introduction of photonic crystals and applications including design and modeling aspects. Photonic crystals are attractive optical materials for controlling and manipulating the flow of light. In particular, photonic crystals are of great interest for both fundamental and applied research, and the two dimensional ones are beginning to find commercial applications such as optical logic devices, micro electro-mechanical systems (MEMS), sensors. The first commercial products involving twodimensionally periodic photonic crystals are already available in the form of photonic-crystal fibers, which use a microscale structure to confine light with radically different characteristics compared to conventional optical fiber for applications in nonlinear devices and guiding wavelengths. The goal of the first volume is to provide an overview about the listed issues.

\title{
How to reference
}

In order to correctly reference this scholarly work, feel free to copy and paste the following:

Kabilan Arunachalam and Susan Christina Xavier (2012). Optical Logic Devices Based on Photonic Crystal, Photonic Crystals - Introduction, Applications and Theory, Dr. Alessandro Massaro (Ed.), ISBN: 978-953-510431-5, InTech, Available from: http://www.intechopen.com/books/photonic-crystals-introduction-applicationsand-theory/optical-logic-devices-based-on-photonic-crystal

\section{INTECH}

open science | open minds

\author{
InTech Europe \\ University Campus STeP Ri \\ Slavka Krautzeka 83/A \\ 51000 Rijeka, Croatia \\ Phone: +385 (51) 770447 \\ Fax: +385 (51) 686166 \\ www.intechopen.com
}

\author{
InTech China \\ Unit 405, Office Block, Hotel Equatorial Shanghai \\ No.65, Yan An Road (West), Shanghai, 200040, China \\ 中国上海市延安西路65号上海国际贵都大饭店办公楼 405 单元 \\ Phone: +86-21-62489820 \\ Fax: +86-21-62489821
}


(C) 2012 The Author(s). Licensee IntechOpen. This is an open access article distributed under the terms of the Creative Commons Attribution 3.0 License, which permits unrestricted use, distribution, and reproduction in any medium, provided the original work is properly cited. 